\title{
Determination of Viscoelastic Properties of Rice Porridge by the Non-Rotational
}

\section{Concentric Cylinder Method}

\author{
Somchai Keawkaika, Kanichi Suzuki and Yoshio Hagura* \\ Graduate School of Biosphere Science, Hiroshima University 1-4-4 Kagamiyama, Higashi-Hiroshima 739-8528, Japan
}

Received June 10, 2009; Accepted October 25, 2009

The viscoelastic properties of Japanese rice porridge were measured using a non-rotational concentric cylinder rheometer. The viscosity and shear modulus of rice porridge exponentially increased with an increase in cooked rice concentration (i.e., $10 \%, 30 \%, 50 \%, 70 \%$ and $97 \%$ rice porridges). A Mooney's type model can be used to reasonably describe the viscosity of rice porridge as a function of the solid volume fraction. Small rice grains made by polishing and grinding produced low viscosity and shear modulus rice porridges. Moreover, the viscosity and shear modulus of rice porridge increased rapidly after cooking owing to an increase in the rice grain volume with water adsorption.

Keywords: rice porridge, viscoelasticity, shear modulus, viscosity, Mooney equation, non-rotational concentric cylinder rheometer

\section{Introduction}

Rice porridge is consumed in many Asian countries, including China, Japan, Indonesia, Thailand, and Vietnam. In some countries, is commonly eaten as breakfast. Moreover, rice porridge is suitable for people who cannot eat hard foods. This includes those who have problems with their digestive system, the elderly whose ability to swallow has declined, and patients who have lost their appetite (Ema and Kainuma, 1996).

Okayu is the type of rice porridge eaten in Japan, and is considerably thicker than rice porridge cooked in other countries. For example, a typical Cantonese-style (China) rice porridge (congee) is made from water and rice grain in the ratio of 12:1, but okayu typically has a ratio of 5:1 (zengayu). In addition, its cooking time is shorter than that of other types of rice porridge; okayu is cooked for about 50 minutes, whereas Cantonese congee is cooked for an hour or more.

In Japan, okayu is well known as a food served to the ill, because it is soft and easily digestible. Okayu is the first solid food served to Japanese infants during the transition period from liquid foods to thicker rice dishes. Japanese rice porridge is classified (according to its ratio of rice to water) as 3-bu kayu, 5-bu kayu, 7-bu kayu and zengayu. 3-bu kayu,

*To whom correspondence should be addressed.

E-mail: hagura@hiroshima-u.ac.jp 5-bu kayu, 7-bu kayu, and zengayu are cooked with ratios of rice to water of $1: 20,1: 10,1: 7.5$, and 1:5, respectively. After cooking, the percentages of cooked rice to water are about $30 \%, 50 \%, 70 \%$, and about 90-100\%, (Ema and Kainuma, 1996). These ratios of rice and water in rice porridge determine the texture and rheological properties, which can be adjusted to suit individual needs.

Most of the literature on the physical and chemical properties of Japanese rice porridge is attributed to Kainuma et al. (Ema and Kainuma, 1996, 1997, 1999, 2000; Kainuma and Fukuda, 2005; Kainuma, 2006a, 2006b). They reported the influences of cooked rice ratio, heating conditions, and elapsed time after cooking on the hardness and adhesiveness of cooked rice grains. The hardness and adhesiveness of cooked rice grain increase with cooked rice ratio from $30 \%$ rice porridge to zengayu (Ema and Kainuma, 1996). In addition, at the same cooked rice ratio, heating conditions (Ema and Kainuma, 1999) and elapsed time after cooking (Ema and Kainuma, 2000) give different physical properties of the rice porridge. However, understanding the texture of rice porridge requires rheological data for the whole rice porridge including cooked rice and continuous phase liquid. Until now, no rheological data on rice porridge have been available, which may be because of the difficulty in measuring with a conventional rheometer.

In the past few decades, the viscoelastic behavior of 
many foods has been studied in terms of dynamic viscoelastic properties. Studies of viscoelastic properties can be done non-destructively if the operation is performed within the linear viscoelastic range. Dynamic viscoelastic measurements have been carried out for many kinds of foods (Rao, 1999; Ross-Murphy, 1995; Rao and Steffe, 1992; Steffe et al., 1986). However, rheological properties of starchy porridges (rice, maize, and wheat) have rarely been studied. Rice porridge contains large solids (rice grains), making it inconvenient to insert a sample into the small gap of a dynamic viscoelastic rheometer.

To solve the problem described above, a novel method employing a non-rotational concentric cylinder (NRCC) rheometer (Suzuki, 1999) was used in this study. This simply structured and easily implemented method allows accurate measurement of the viscosity $(\mu)$ and shear modulus $(G)$ of liquid foods. The space between the plunger and the measuring cup is sufficiently large for use with liquid foods containing large particles, such as rice granules. Previously, the NRCC method has been use for studying viscoelastic properties of foods such as mayonnaise, gelatinized starch dispersion, and ketchup (Suzuki, 1999; Suzuki et al., 2007; Keawkaika et al., 2007).

In the present study, the NRCC method was used to study the viscoelastic properties of Japanese rice porridge of various rice contents. The influences of solid (rice granule) concentration and rice grain size on viscoelastic properties of rice porridge were studied. In addition, parameters affecting viscoelastic properties of a sample after cooking were also investigated.

\section{Brief Background}

Viscoelasticities determination by NRCC method Figure 1 shows a theoretical scheme of the method employed in this paper. The theoretical derivation of the measurement system has been presented by Suzuki et al. (2007) in a previous pa-

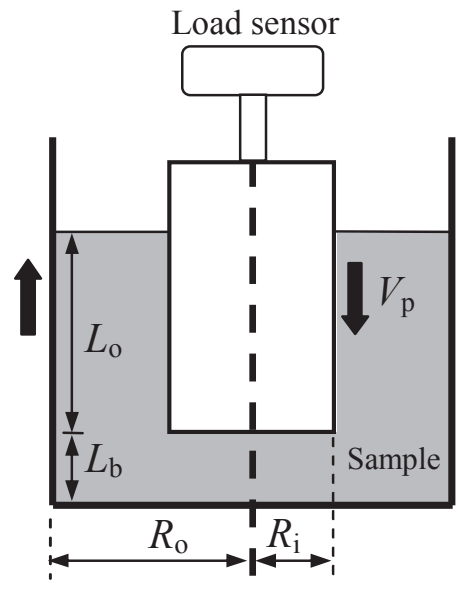

Fig. 1. Non-rotational concentric cylinder rheometer. per. A plunger (radius: $R_{\mathrm{i}}$ ) is initially dipped at a distance, $L_{0}$, in the liquid sample, which is in a cup (radius: $R_{\mathrm{o}}$ ). The initial distance between the plunger's bottom and the cup bottom is $L_{\mathrm{b}}$. The cup is moved upward or the plunger is moved downward for a distance $\Delta L$ at a constant speed, $V_{\mathrm{p}}$.

The NRCC method seems to employ a structure similar to that of the back-extrusion method, proposed by Morgan et al. (1979). Moreover, Thermal Scanning Rigidity Monitor (TSRM) was also developed based on the back-extrusion method under the temperature controlled condition ( $\mathrm{Wu}$ et al., 1985a, 1985b). However, the measurement by the backextrusion method or the TSRM was done by downward movement of the plunger into the sample cup, which consequently pushes the sample to flow upward. Then, the viscosity could be calculated from the loaded stress as functions of flowing time and geometrical dimensions of the plunger and the sample cup. However, for the viscoelastic liquid which has both viscous and elastic parts, the back-extrusion method cannot differentiate the two types of stresses, but detects all stresses as the viscous stress. Meanwhile, the theory of the NRCC method promises an ability to detect viscosity and shear modulus of liquid sample separately from the total force (Suzuki et al., 2007). For measurement in the NRCC method, the cup containing the liquid sample moves upward at a constant speed over a very small distance $(0.1$ $0.2 \mathrm{~mm}$ ). As the result, the plunger, which stays still inside the cup, has downward motion relative to the sample, and the flow of the sample is very short. Moreover, the measurement time in this method is very short. Viscosity and shear modulus can be detected just at the starting time of the cup movement.

Suzuki (1999) has shown the accurate viscosities of glycerol solution and sucrose solution measured by the NRCC method and compared them with the literature values (Lange, 1967). Moreover, the viscosities of mayonnaise, ketchup, salad dressing, and gum arabic measured by the NRCC method were the same as those measured by the Stresstech rheometer (DAR50, Rheologica Instrument, A.B.). The NRCC method can also measure viscosity and shear modulus of viscoelastic liquid materials simultaneously. The theory and experimental results are found elsewhere (Suzuki, 1999; Suzuki et al., 2007; Keawkaika et al., 2007).

Estimation of the viscosity of rice porridge For a concentrated suspension of rigid spherical particles, Palierne (1990) has shown the relationship between complex shear modulus $\left(G^{*}\right)$ and dispersed phase volume fraction to be

$$
G^{*}=G_{C}^{*}\left[\frac{1+\frac{3}{2} \phi}{1-\phi}\right]
$$


where $\phi$ is the dispersed phase volume fraction and $G_{C}^{*}$ is the complex shear modulus of the continuous phase, defined as

$$
G^{*}=G^{\prime}+j G^{\prime \prime}
$$

where $G^{\prime}$ is the storage modulus and $G^{\prime \prime}$ is the loss modulus, and $j$ is a complex number. Moreover, Eq. 1 can be written in terms of the complex shear viscosity $\left(\mu^{*}\right)$ as

$$
\mu^{*}=\mu_{C}^{*}\left[\frac{1+\frac{3}{2} \phi}{1-\phi}\right]
$$

where $\mu_{c}^{*}$ is the complex shear viscosity of the continuous phase. Note that $\mu^{*}=G^{*} / j \omega$, where $\omega$ is angular frequency.

Generally, Eq. 1 is valid for moderately concentrated suspensions. For high dispersed phase concentrations, Eq. 1 underpredicts the values of the storage and loss moduli.

For a concentrated emulsion of deformable droplets, Palierne (1990) has shown that the complex shear modulus can be defined as

$$
G^{*}=G_{C}^{*}\left[\frac{1+\frac{3}{2} \phi H}{1-\phi H}\right]
$$

where

$$
H=\frac{2\left[\left(G_{d}^{*}-G_{C}^{*}\right)\left(19 G_{d}^{*}+16 G_{C}^{*}\right)+(4 \sigma / R)\left(5 G_{d}^{*}+2 G_{C}^{*}\right)\right]}{\left[\left(2 G_{d}^{*}+3 G_{C}^{*}\right)\left(19 G_{d}^{*}+16 G_{C}^{*}\right)+(40 \sigma / R)\left(G_{d}^{*}+G_{C}^{*}\right)\right]}
$$

where $G_{d}^{*}$ is a complex modulus of dispersed phase, $\sigma$ is interfacial tension, and $R$ is droplet radius.

However, Eq. 4 is generally valid for moderately concentrated emulsions. For concentrated emulsions in which aggregation and flocculation of droplet occur, Eq. 4 underpredicts the storage and loss moduli of samples.

Numerous researchers (Krieger and Dougherty, 1959; Metzner, 1985; Kitano et al., 1981; Phan-Thien and Pham, 1997) have proposed empirical equations to describe viscoelasticities of solid particle suspensions. Most empirical equations are generally valid in either moderate or highly concentrated emulsions. This is due to the occurrence of aggregation and flocculation in highly concentrated systems. Especially, in food dispersions, aggregation and flocculation occur when the dispersed phase varies from low to high concentration, which causes unpredictable viscoelasticity in these systems. Recently, Pal (1996) proposed the following modified Mooney equations to describe the relative viscosity and viscoelastic moduli of concentrated polymer-thickened emulsions,

$$
\begin{aligned}
& \mu_{r}=\exp \left[\frac{K_{1} \phi}{1-K_{2} \phi}\right] \\
& G^{\prime} r=\exp \left[\frac{K_{1} \phi}{1-K_{2} \phi}\right] \\
& G_{r}^{\prime \prime}=\exp \left[\frac{K_{1} \phi}{1-K_{2} \phi}\right]
\end{aligned}
$$

where $\mu_{r}$ is relative viscosity (ratio of emulsion viscosity to continuous phase viscosity at the same shear rate), $G_{r}^{\prime}$ and $G^{\prime \prime}{ }_{r}$ are relative dynamic moduli (ratio of emulsion modulus to continuous phase modulus at the same frequency), and $K_{1}$ and $K_{2}$ are adjustable parameters. Pal (1996) showed that $K_{1}$ and $K_{2}$ change with shear rate and frequency. The results show that these equations adequately describe dynamic viscoelastic moduli of emulsions for oil volume fractions up to 0.77. Eq. 7 and Eq. 8 were used to predict the viscoelastic moduli of multiphase dispersions (Pal, 2000).

Rice porridge can be defined as a suspension of solid in a liquid medium. At low content of cooked rice, rice grains fully swell and disperse into the continuous phase. Similar to oil droplets in emulsion, rice grains deform and pack into a compacted system at higher concentration, when available space is limited. In this work, the modified Mooney equations (Eq. 6) were used to predict the viscosity of rice porridge for various solid volume fractions.

\section{Materials and Methods}

Materials Rice porridge was prepared from grains of $\mathrm{Ko}-$ shihikari, a non-waxy japonica-type rice cultivar (Oryza sativa var. japonica) harvested in 2006 from Niigata prefecture.

Rice porridge preparation Rice porridge was prepared by cooking the dispersed phase and continuous phase separately, and then mixing them together. For the dispersed phase, zengayu was used, which was prepared using an electric okayu maker (EG-BA06, Zojirushi Mahobin, Tokyo, Japan) purchased from a local market, and its water to rice ratio was $5: 1$ by volume. Zengayu was cooked at a temperature of about $95^{\circ} \mathrm{C}$ for $60 \mathrm{~min}$.

For the continuous phase, the original intent was to use отоуи. Отоуи is a rice porridge that can be prepared by cooking water and rice in a ratio of 10:1 for about 1 hour. The rice porridge thus obtained is filtered through a screen that has a screening size smaller than the cooked rice particle size. Отоуи is the obtained liquid portion. However, in this study, the continuous phase was prepared using gelatinized rice flour solution instead, because of the difficulty in controlling the viscosity of omoyu. Rice flour made from 100\% rice grain was purchased from a local market. A $4 \mathrm{w} / \mathrm{w} \%$ 
gelatinized rice flour solution was used as continuous phase and was prepared by heating the solution to $90^{\circ} \mathrm{C}$ for $30 \mathrm{~min}-$ utes by means of a hot plate. The solution was gently stirred during heating to prevent the aggregation and agglomeration of starch granules. The viscosity at this concentration was confirmed to be nearly the same as that of original omoyu.

Rice porridge samples were prepared by mixing zengayu with the continuous phase at various ratios (i.e., zengayu: continuous phase $=1: 9,3: 7,5: 5,7: 3,10: 0)$. The final solid volume fractions $(\phi)$ of samples were $0.1,0.3,0.5$, and 0.7 , respectively. Since some water still remained in zengayu, the solid volume fraction was assumed to be 0.97 . Therefore, the samples were called $10 \%, 30 \%, 50 \%, 70 \%$, and $97 \%$ rice porridges, respectively.

To study the effect of rice grain size on viscosity, three types of rice grains were used as the samples: whole rice grain, 20\% polished rice grain, and ground rice grain. A compact testing mill, HS-4 (Chiyoda Engineering, Tokyo, Japan), was used to polish the rice grain to a smaller size. $20 \%$ polished rice grain was polished at $1800 \mathrm{rpm}$ to $80 \mathrm{w} /$ $\mathrm{w} \%$ of its original weight. Ground rice grain was prepared by crushing rice grains into small pieces using an analytical mill, MF10 (IKA-WERKE, Germany). Rotational speed was $3000 \mathrm{rpm}$ and sieve size was $3.0 \mathrm{~mm}$. The obtained ground rice particles were sieved through screening sieves for which sieve sizes were $2.36 \mathrm{~mm}, 1.70 \mathrm{~mm}, 1.18 \mathrm{~mm}$, and $0.85 \mathrm{~mm}$. The particles passing through the $2.36 \mathrm{~mm}$ sieve and retained over the $1.70 \mathrm{~mm}$ sieve were used as the ground rice sample.

The average widths of samples were $4.8 \mathrm{~mm}$ (whole rice grain), $4.1 \mathrm{~mm}$ ( $20 \%$ polished rice grain), and around $1.70-2.36 \mathrm{~mm}$ (ground rice grain).

Swelling index of rice grain After rice porridge was made, 30 grains of cooked rice porridge were put into a 25 $\mathrm{mL}$ measuring cylinder, which contained $15 \mathrm{~mL}$ of water $\left(25^{\circ} \mathrm{C}\right)$. The increase in volume was observed by the change in the water level. The rice grain swelling ratio, therefore, was calculated from the volume of cooked rice to the volume of uncooked rice.

Viscoelastic measurement Static viscoelasticity was measured by means of a non-rotational concentric cylinder rheometer (NRCC-Visco-PRO, Sun Scientific Co., Tokyo, Japan). Figure 1 shows the measuring apparatus used in the NRCC method. Plunger no. $1\left(R_{\mathrm{i}}=49.09 \mathrm{~mm}, R_{\mathrm{o}}=50.07\right.$ $\mathrm{mm}$, and $\left.\kappa=R_{\mathrm{i}} / R_{\mathrm{o}}=0.98\right)$ of the apparatus was used; where $L_{0}=51.52 \mathrm{~mm}$, and $L_{\mathrm{b}}=15.00 \mathrm{~mm}$. The plunger velocity $\left(V_{\mathrm{p}}\right)$ ranged from $10 \mathrm{~mm} / \mathrm{min}$ to $80 \mathrm{~mm} / \mathrm{min}$, and the plunger movement distance $(\Delta L)$ ranged from $0.1 \mathrm{~mm}$ to $0.2 \mathrm{~mm}$. Measurement time was within $2 \mathrm{~s}$. The dispersed phase and continuous phase were mixed immediately after the dispersed phase (zengayu) was cooked, and the temperature of both phases after mixing varied between 85 and $87^{\circ} \mathrm{C}$. The samples were then quickly transferred to the measuring cup. To prevent the influence of modification in structure due to sample handling and while loading the sample in a measuring cup, samples were stored in the cup for $10 \mathrm{~min}$ before measurement. The samples cannot be stored in excess of 10 min, because the temperature is too high and the water may evaporate. All viscoelastic measurements were performed at a temperature of $85^{\circ} \mathrm{C}$, which was controlled using a water circulation system. During the viscoelastic measurement, samples were covered by a thin layer of oil to prevent water loss. The measurements were performed five times, and the average values were used as the results.

\section{Results and Discussion}

Effect of dispersed phase concentration Rice porridges with various rice contents were prepared by changing the cooked rice to water ratio, which gave $10 \%, 30 \%, 50 \%$, $70 \%$, and $97 \%$ rice porridges. Figure 2 shows the plots of viscosity $(\mu)$ and shear modulus $(G)$ versus shear rate for different cooked rice concentrations. At low cooked rice concentrations ( $0 \%$ to $30 \%)$, the samples show a small shear thinning behavior; viscosity decreases with increasing shear rate. As the cooked rice content increases, the shear thinning
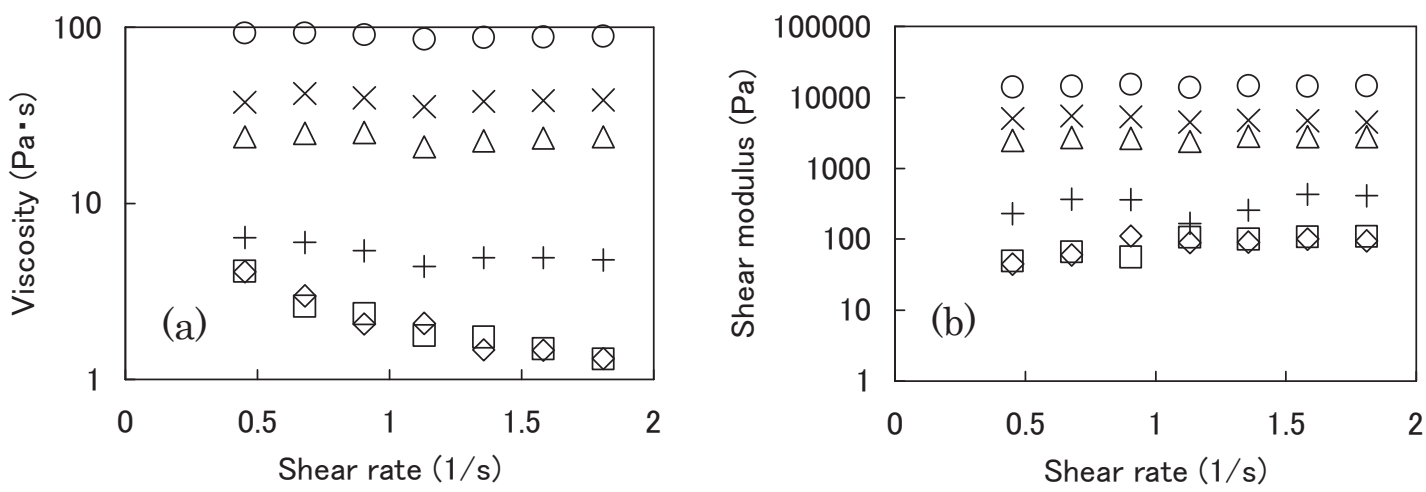

Fig. 2. Viscosity (a) and shear modulus (b) of $0 \%(\diamond), 10 \%(\square), 30 \%(+), 50 \%(\triangle), 70 \%(\times)$ and $97 \%(\bigcirc)$ rice porridges (whole rice grain). 

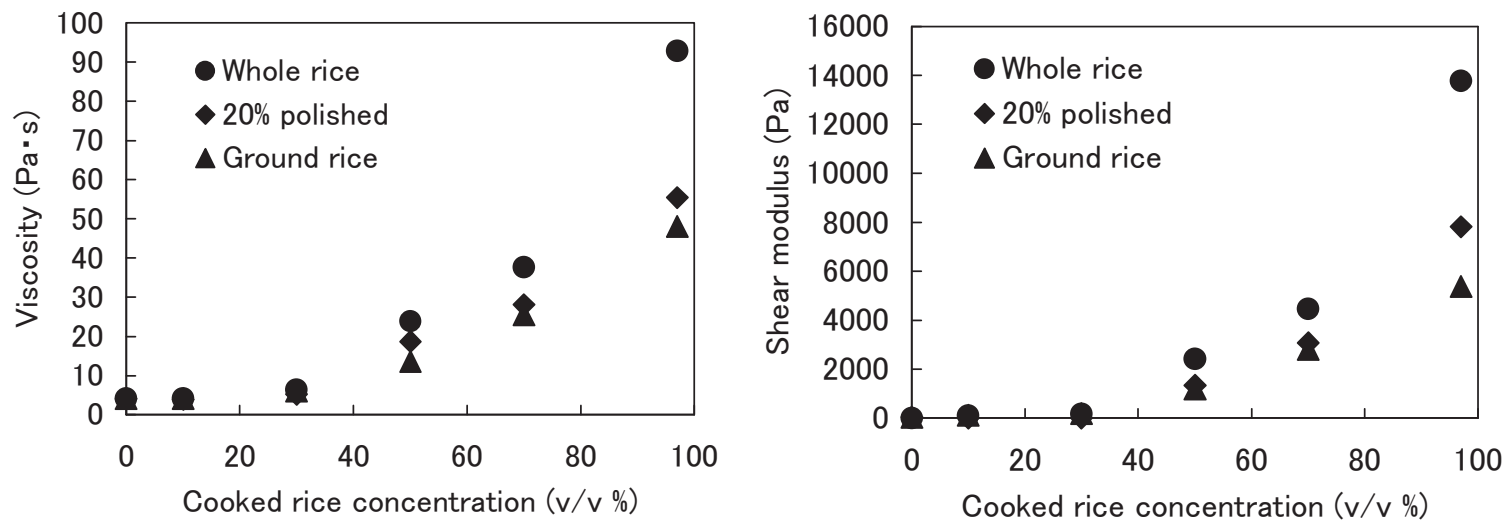

Fig. 3. Viscosity (a) and shear modulus (b) of rice porridges with different types of rice grains, measured at a shear rate of $0.451 / \mathrm{s}$.

behavior seems to disappear. On the other hand, shear modulus shows shear rate independence over the observed range.

The viscosity and shear modulus of the samples have almost the same trend, in that they increase exponentially with cooked rice concentrations (Fig. 3). The viscosities and shear moduli of $0 \%$ to $30 \%$ rice porridges were extensively low. At concentrations higher than $30 \%$, the viscosity and shear modulus increased rapidly with an increase in cooked rice concentration. This can be attributed to an increase in the contact among rice grains as the cooked rice concentration increased. As a result, samples become more viscous. However, at higher concentration, as the system reaches a close packing concentration, the further addition of cooked rice causes the deformation of rice grains.

It is difficult to explain why $\mu$ and $G$ have the same trend when the cooked rice concentration increases. One possibility is to consider the cooked rice to be the rigid part of the system and the liquid to be the viscous part of the system. The high rice concentration gives rise to the rigidity of the system, which causes the high shear moduli of the samples. However, from another point of view, the increase in rice concentration results in more concentrated systems. As a result, samples become more viscous.

From the results shown above, the viscosity of rice porridge is considered to correlate with the solid dispersed volume fraction, which refers to cooked rice volume fraction. Therefore, the Mooney type equation (Eq. 6) was used to describe the influence of dispersed phase (cooked rice) volume fraction $(\phi)$ on the viscosity of rice porridge. Figure 4 compares the experimental values of relative viscosity with the values calculated from the Mooney type equation. As can be seen from this figure, the Mooney type equation describes the viscosity as a function of $\phi$ reasonably well.

The result given above confirms that the viscosity of rice porridge can be estimated from the amount of cooked rice. As can be seen from Figure 3 (a), the viscosity of $97 \%$ whole rice porridge was more than three times that of $50 \%$ whole rice porridge. These data can be used for adjusting the texture of porridge to make it appropriate for patients with different levels of swallowing and chewing ability. However, it should be noted that at low volume fractions the calculated values are higher than experimental values, possibly due to sedimentation of rice grains.

Effect of rice grain size As shown in Figure 3, the viscosity and shear modulus of samples decrease with decreasing rice grain size. The average widths of rice grain were 4.8 $\mathrm{mm}$ (whole rice grain), $4.1 \mathrm{~mm}$ ( $20 \%$ polished rice grain) and around 1.70-2.36 mm (ground rice grain). The whole rice grain (rod shape) had very high values of viscosity and shear modulus. Especially for $97 \%$ rice porridge, $\mu$ and $G$ values of whole rice grain were about twice those of ground rice grain. The polished and ground rice grains were softened after cooking, which may result in lowered viscosity and shear modulus.

As can see from Figure 4, the Mooney type equation describes the viscosity of rice porridges made from different sized rice grains reasonably well. Moreover, the constants $K_{1}$ and $K_{2}$ in the Mooney type equation varied with the rice grain size. Table 1 summarizes the values of the constants for the data shown in Figure 4. For colloidal dispersions with

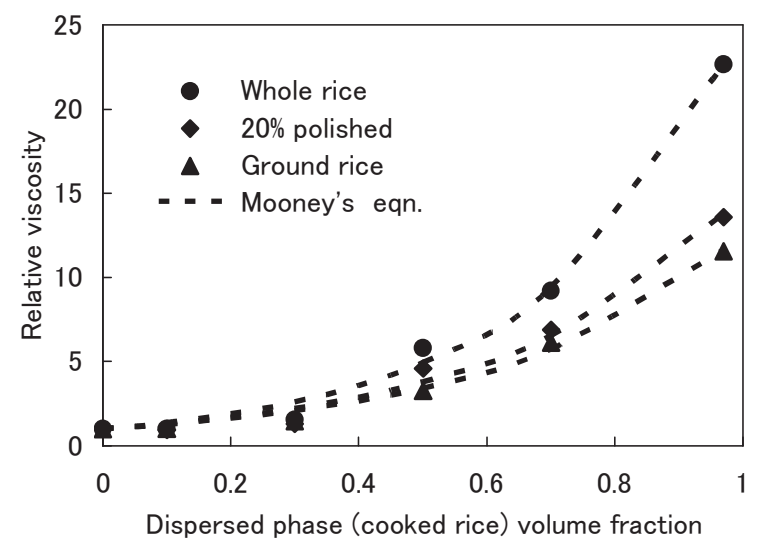

Fig. 4. Correlation of relative viscosity data for rice porridges with different types of rice grains, measured at a shear rate of $0.451 / \mathrm{s}$. 
Table 1. Constants $K_{1}$ and $K_{2}$ in the Mooney type equation for rice porridges.

\begin{tabular}{lcc}
\hline Sample & $K_{1}$ & $K_{2}$ \\
\hline Whole rice & 3.176 & 0.013 \\
20\% polished rice & 2.630 & 0.028 \\
Ground rice & 2.411 & 0.048 \\
\hline
\end{tabular}

particle size ranging between about one nanometer and one micrometer (Schramm, 2005), the constant $K_{2}$ decreases with increasing particle size. The constant $K_{1}$ is 2.76 or 2.50 and the constant $K_{2}$ varies between 1.00 and 1.47 (Parkinson et al., 1970; Sherman, 1970). Unfortunately, the rice grain sizes used in this study were much larger than those colloidal dispersions. Thus, comparing the constants with those studies is difficult. However, these results show that $K_{2}$ decreases with increasing rice grain size.

Effect of elapsed time after cooking Rice porridge is known to thicken rapidly after cooking. Therefore, a sample was kept at constant temperature $\left(85^{\circ} \mathrm{C}\right)$ to study the influence of storage time on viscoelasticity. Figure 5 shows changes in viscosity and shear modulus as functions of elapsed time for the $50 \%$ and the $97 \%$ rice porridges after cooking. It should be noted that at high temperature, solid volume fraction may increase due to water evaporation. Therefore, to prevent the effect of water evaporation on viscoelasticity, samples were covered by a thin layer of oil. Moreover, the experiment for the $97 \%$ rice porridge was performed for only one hour because of difficulty in preventing water evaporation from this porridge after one hour at high temperature.

As can see from Figure 5, the viscosity and shear modulus of both samples gradually increase with time. The increase in viscosity and shear modulus of a sample is perhaps mainly responsible for the increase in volume of cooked rice grains. The swelling ratios of rice grains increased gradually during 60 min observation time (Fig. 6). The greater the rice grain volume, the higher the solid volume fraction of the sys-

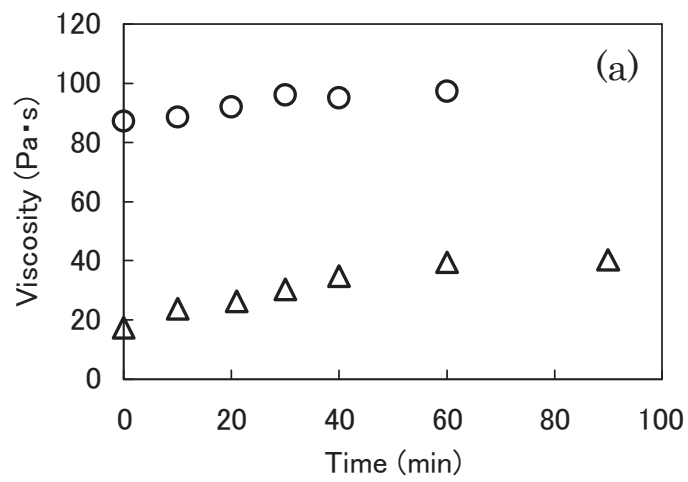

tem, and the higher the viscosity and shear modulus of the sample.

There should be limitation of the swelling ratios once the rice grains fully swell and the water in the system is exhausted. The viscosity and shear modulus of the $97 \%$ rice porridge increase rapidly for the first 30 min after cooking. Afterwards, the viscosity and shear modulus seem to be constant, suggesting that the rice grains cannot swell further due to the lack of water in the system. Ema and Kainuma (2000) showed an increase of hardness and adhesiveness of $70 \%$ and the $97 \%$ rice porridges with time when samples were stored at $60^{\circ} \mathrm{C}$ and $80^{\circ} \mathrm{C}$.

The increase in the viscosity of continuous phase also influences the rice porridge viscoelasticity. A small increase in viscosity of continuous phase was observed when the sample was stored at $85^{\circ} \mathrm{C}$ for one hour (Fig. 6). During the heating period, starch granules absorb the water and swell, causing the volume fraction to rise and finally reach a maximum value. With further heating, the granule ruptures and releases amylose, and this contributes to the increase in viscosity of the continuous phase (Rao, 1999).

During gelatinization of starch dispersions, starch granules swell several times in size as temperature rises. A study on starch gelatinization using the dynamic viscoelastic method revealed that the storage modulus $\left(G^{\prime}\right)$ of rice starch dispersion increased to a maximum at a temperature of $72.4^{\circ} \mathrm{C}$ (Singh et al., 2003). The storage modulus then decreased owing to the physical breakdown of starch granules (Eliasson, 1986). However, in the present study, the samples are in the form of rice grains that contain starch granules inside, and this might lead to the difference in the behaviors of starch granules. In particular, most starch granules in rice grains could not swell freely to their maximum volume during the heating period $\left(95^{\circ} \mathrm{C}, 60 \mathrm{~min}\right)$. Consequently, the rice granules continue swelling during the storage period, resulting in increases in viscosity and shear modulus of the sample. Furthermore, the structure breakdown of the starch granules

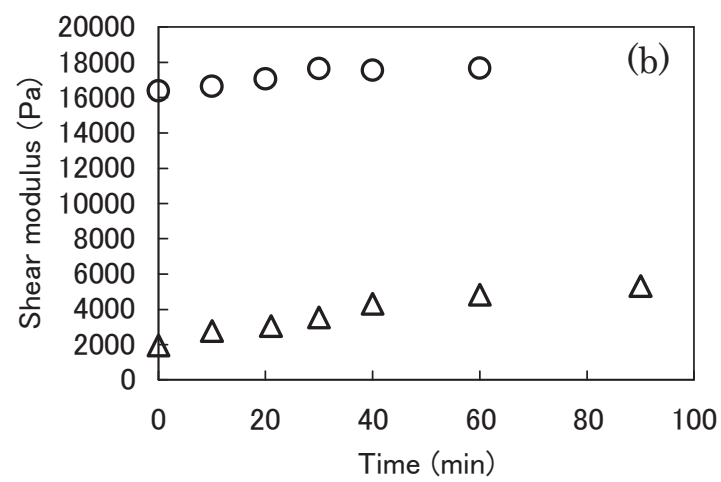

Fig. 6. Viscosity (a) and shear modulus (b) of the $50 \%(\triangle)$ and $97 \%(\bigcirc)$ rice porridges as a function of elapsed time at $85^{\circ} \mathrm{C}$, measured at a shear rate of $1.131 / \mathrm{s}$. 
is delayed, since no decreases in viscosities and shear moduli of rice porridges were observed.

Effect of storage temperature During the cooling of gelatinized starch, the viscosity of the starch paste increases due to retrogradation associated with swollen granules, fragments of swollen granules, and dispersed starch molecules (Singh et al., 2003). Moreover, amylose forms double-helical associations of 40-70 glucose units (Jane and Robyt, 1984) and amylopectin crystallization occurs with the formation of the outermost short branches (Ring et al., 1987).

Figure 7 shows temperature changes for the $50 \%$ and $97 \%$ rice porridges with natural cooling. The two samples have almost the same temperature curves for $90 \mathrm{~min}$. Figure 8 shows the influences of temperature on the viscosities and shear moduli of both samples. In the case of $97 \%$ rice porridge, only a small amount of water remained, and a rapid increase in the viscosity and shear modulus could be observed as the temperature decreased. The increases in viscosity and shear modulus are possibly caused by the retrogradation effect of the gelatinized starch during the cooling period. It should be noted that after one hour, the viscosity and shear modulus of the $97 \%$ rice porridge decreased because of water evaporation. Due to the loss of almost all of water, the rice porridge became crumbly and lost its stickiness.

However, in the case of the $50 \%$ rice porridge, a large amount of water still remained at the end of the experiment, and the viscosity and shear modulus of a sample during cooling (Fig. 8) had almost the same values as a sample at constant temperature (Fig. 5) until $90 \mathrm{~min}$. These results demonstrate that when water remains in the system, the swelling of rice grains is the main factor for the increase in viscosity and shear modulus of rice porridge.

In fact, in many cases, rice porridge is not consumed immediately after cooking. For instance, after preparing rice porridge, time is taken before serving. If the rice porridge has high water content or the temperature after cooking is high, the viscoelasticity of the rice porridge will increase due to
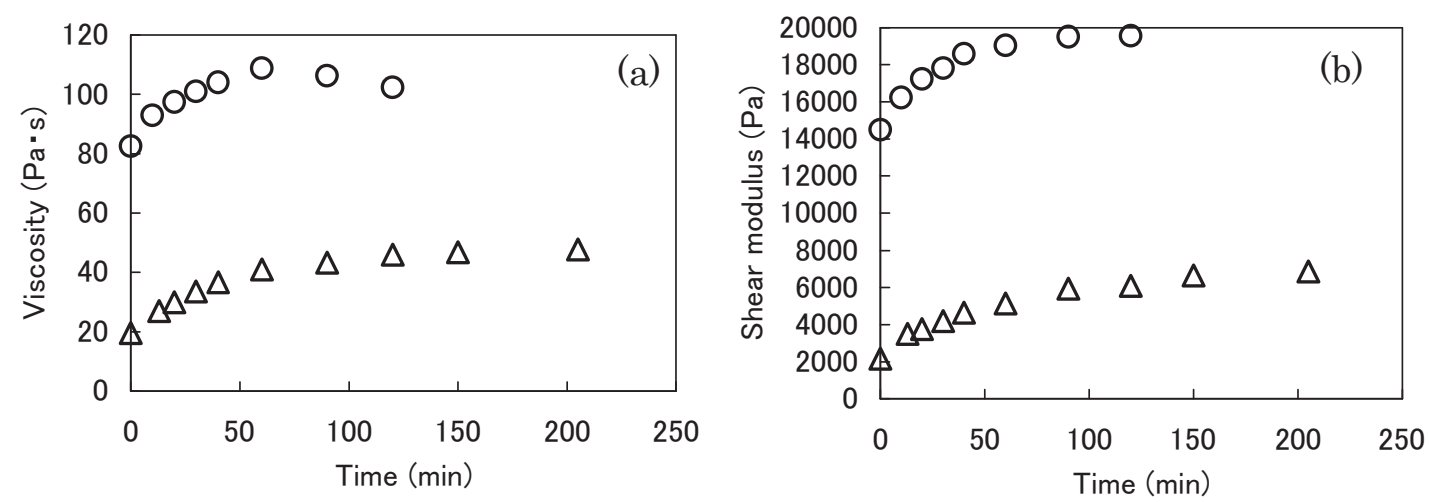

Fig. 8. Viscosity (a) and shear modulus (b) of the $50 \%(\triangle)$ and $97 \%(\bigcirc)$ rice porridges with natural cooling, measured at a shear rate of $1.131 / \mathrm{s}$. 
standing of their texture properties. Therefore, the rheological data obtained by this method can be used for people who have problems with their digestive systems and the elderly whose chewing and swallowing abilities have declined.

\section{References}

Eliasson, A.C. (1986). Viscoelastic behavior during the gelatinization of starch. I. Comparison of wheat, maize, potato and waxybarley starches. J. Texture Studies, 17, 253-265.

Ema, S., Kainuma, Y. (1996). The study of Japanese rice gruel cooking: the quality of rice gruel made of Japanese rice and imported rice. Journal of Home Economics of Japan, 47, 29-36.

Ema, S., Kainuma, Y. (1997). The study of Japanese rice gruel cooking (Part 2): effect of heating conditions on the properties of "Zengayu". Journal of Home Economics of Japan, 48, 391-398.

Ema, S., Kainuma, Y. (1999). The study of Japanese rice gruel cooking (Part 3): effect of cooking pot material and heating conditions on the properties of "Zengayu". Journal of Home Economics of Japan, 50, 341-347.

Ema, S., Kainuma, Y. (2000). The study of Japanese rice gruel cooking (Part 4): effect of elapsed time after cooking and keeping warm conditions on the properties of rice gruel. Journal of Home Economics of Japan, 51, 571-578.

Jane, J.L., Robyt, J.F. (1984). Structure studies of amylose V complexes and retrograded amylose by action of alpha amylase, a new method for preparing amylodextrins. Carbohydrate Research, 132, 105-118.

Kainuma, Y., Fukuda, Y. (2005). Preparation method for rice gruel made from cooked rice of long-stored rice. Journal of Home Economics of Japan, 56, 215-222.

Kainuma, Y. (2006a). Properties of the rice gruels prepared from cooked rice with varying ratios of water to raw rice. Journal of Home Economics of Japan, 57, 199-207.

Kainuma, Y. (2006b). Refrigeration condition for the preservation of rice gruel. Journal of Home Economics of Japan, 57, 785-792.

Keawkaika, S., Hagura, Y., and Suzuki, K. (2007). Comparison of the viscoelastic properties of fluid foods measured by the nonrotational concentric cylinder method with those by the dynamic oscillatory method. Jpn. J. Food Eng., 8, 155-162.

Kitano, T., Kataoka, T., and Shirota, T. (1981). An empirical equation of the relative viscosity of polymer melts filled with various inorganic fillers. Rheol. Acta, 20, 207-209.

Krieger, I.M., Dougherty, T.J. (1959). A mechanism for non-Newtonian flow in suspensions of rigid spheres. Trans. Soc. Rheol., 3, 137-152.

Lange, N.A. (1967). Handbook of Chemistry, $10^{\text {th }}$ ed. McGraw-Hill, New York, NY.

Metzner, A.B. (1985). Rheology of suspensions in polymeric liquids. J. Rheol., 29, 739-775.

Morgan, R.G., Suter, D.A. and Sweat, V.E. (1979). Mathematical analysis of a simple back-extrusion rheometer. American Society of Agricultural Engineering, 79, 6001.

Pal, R. (1996). Viscoelastic properties of polymer-thickened oil-inwater emulsions. Chem. Eng. Sci., 51, 3299-3305.

Pal, R (2000). Linear viscoelastic behavior of multiphase dispersions. J. Colloid Interface Sci., 232, 50-63.

Palierne, J.F. (1990). Linear rheology of viscoelastic emulsions with interfacial tension. Rheol. Acta, 29, 204-214.

Parkinson, C., Matsumoto, S., and Sherman, P. (1970). The influence of particle-size distribution on the apparent viscosity of non-Newtonian dispersed systems. J. Colloid Interface Sci., 33, 150-160.

Phan-Thien, N., Pham, D.C. (1997). Differential multiphase models for polydispersed suspensions and particulate solids. J. NonNewtonian Fluid Mech., 72, 305-318.

Rao, M.A. (1999). Rheology of fluid and semisolid foods. Maryland: Aspen Publishers.

Rao, M.A., Steffe, J.F. (1992). Viscoelastic properties of foods. Essex, England: Elsevier Science Publishers.

Ring, S.G., Collona, P., Panson, K. J., Kalicheversky, M. T., Miles, M.J., Morris, V. J., and Oxford, P.D. (1987). The gelation and crystallization of amylopectin. Carbohydrate Research, 162, 277-293.

Ross-Murphy, S. B. (1995). Rheological characterisation of gels. J. Texture Studies, 26, 391-400.

Schramm, L.L. (2005). Emulsions, Foams, and Suspensions: Fundamentals and Applications. Weinheim: WILEY-VCH Verlag GmbH \& Co. KGaA.

Sherman, P. (1970). Industrial Rheology. New York: Academic Press.

Singh, N., Singh, J., Kaur, L., Sodhi, N. S., and Gill, B.S. (2003). Morphological, thermal and rheological properties of starches from different botanical sources. Food Chem., 81, 219-231.

Steffe, J.F., Mohamed, I.O., and Ford, E.W. (1986). Rheological properties of fluids foods: data compilation. In M.R. Okos (Ed.), Physical and chemical properties of food. Michigan :American Society of Agricultural engineer,1-13pp.

Suzuki, K. (1999). A novel method for evaluating viscosity and viscoelasticity of liquid foods by a non-rotational concentric cylinder setup. Nippon Shokuhin Kagaku Kogaku Kaishi, 46, 657-663.

Suzuki, K., Imaoka, K., Keawkaika, S., and Hagura, Y. (2007). Two-element model analysis of the viscoelastic behavior of liquid food materials by means of the non-rotational concentric cylinder method. Jpn. J. Food Eng., 8, 73-80.

Wu, M.C., Lanier, T.C. and Hamann, D.D. (1985a). Rigidity and Viscosity Changes of Croaker Actomyosin During Thermal Gelation. J. Food Sci., 50, 14-19.

Wu, M.C., Hamann, D.D. and Lanier, T.C. (1985b). Rheological and Calorimetric Investigations of Starch-fish Protein Systems during Thermal Processing. J. Texture Studies, 16, 53-74. 